\title{
THE XML AND SEMANTIC WEB: A SYSTEMATIC REVIEW ON TECHNOLOGIES
}

\author{
Mohamed Alloghani ${ }^{1,2}$, Dhiya Al-Jumeily ${ }^{1}$, Abir Hussain ${ }^{1}$, Ahmed J. Aljaaf ${ }^{1,3}$, \\ Jamila Mustafina ${ }^{4}$, Mohammed Khalaf ${ }^{5}$ and Sin Y. Tan ${ }^{1}$ \\ ${ }^{1}$ Liverpool John Moores University, Liverpool L3 3AF, UK \\ ${ }^{2}$ Abu Dhabi Health Services Company (SEHA), Abu Dhabi, UAE \\ ${ }^{3}$ Centre of Computer, University of Anbar, Iraq \\ ${ }^{4}$ Kazan Federal University, Kazan, Russia \\ ${ }^{5}$ Almaaref University College, Anbar, Ramadi, Iraq
}

\begin{abstract}
The web and associated data have been around before the advent of big data and its related concepts. The semantic web refers to a Web of Data consisting of all sorts of data types and forms. In the context of Web of Data, many organizations prefer Linked Data formats for data publication, sharing and usage based on semantic web (SW) technologies. Conversely, the Extensible Markup Language (XML) is the commonly used data exchange format that the world wide web (WWW) uses today. Furthermore, innumerable international data exchange formats including Moving Picture Experts Group-7 (MPEG-7) and Institute of Electrical and Electronics Engineers Learning Object Metadata (IEEE LOM) rely on XML schema, and consequently, the web has a massive volume of XML format-based datasets. Of interest is the difference between SW and XML data models, frameworks as well as the language for implementing data queries. Hence, it is necessary to pursue interoperability and integration technologies for bridging the gap between SW and XML frameworks to ensure seamless data handling and retrieval in the web. The systematic survey used Preferred Reporting Items for Systematic Reviews and Meta-Analyses (PRISMA) methodology alongside DistillerSR to review articles addressing the interoperability and integration of XML and SW. The advanced search query focused on the article title, abstract, full text, and keywords that are related to the XML and SW technologies.
\end{abstract}

\section{KEYWORDS}

XML, WWW, XML Schema, Semantic Web (SW), SPARQL2XQuery, Xs2OWL

\section{INTRODUCTION}

One of the key features of data, especially those that are voluminous is the format and its compatibility with different analytical and information retrieval platforms. An exemplary example is the Web of Data, which unfortunately have several formats and standards depending on the industry, organization, and the purpose or strategic objective of the dataset. However, in the context of Web of Data, most organisations gravitate towards Linked Data because they rely on Semantic Web (SW) techniques for data publication with an expectation that target audience uses SPARQL to search, retrieve, and use the published data. It is imperative to note that SPARQL is a data query language without internal inferences and it retrieves data presented in a specific model or data format (Batra, Sachdeva, \& Bhalla, 2018). However, Extensible Markup Language (XML) is the dominant data format used in the web, while most of the other international standards such as MPEG-7, IEEE LOM and MPEG-21 among others use XML schema making XML datasets the largest in the context of Web of Data.

Notably, semantic web (SW) is an extension of world wide web, and it qualifies as a Web of Data. Some of the semantic web technologies commonly used in data include but not limited to Resource Description Framework (RDF), Web Ontology Language (OWL), Simple Knowledge Organization System (SKOS), and SPARQL(Anonymous, 2014; Jing, Kay, Marley, \& Hardiker, 2014; Scharffe, Zamazal, \& Fensel, 2014). Despite the difference between SW and XML data format, applications and services must coexist within the 
web framework, and as such their interoperation is critical to the existence of applications with unfettered access to legacy systems (Zemmouchi-Ghomari, 2018). Both SW and XML schema and its related technologies will remain critical for the effectiveness of Web of Data, especially from the perspective of Linked Data.

Linked Data is the foundation of SW because it permits large scale integration of data on the web. The web contains all sorts and types of data including qualitative, quantitative, and visual data (Grossi, Romei, \& Turini, 2017; Li, Martínez, \& Rubio, 2017; Robson, 2016; Soylu et al., 2017). It is imperative to note that the format of these categories of data differs as well as the query language used to fetch them. It is important to note that platforms and retrieval forms are also different, but regardless of these differences, the categories and data types have a complex linkage that supports accessibility from single queries (Naumenko, Nikitin, Terziyan, \& Zharko, 2005; Umar, 2005). That is, an implemented query returns search results that consist of images, videos, and texts concurrently, although most search engines present the results using relevant categories.

\section{MOTIVATION AND SCOPE}

The proliferation of technologies such as the Internet of Things, blogging, microblogging, and social media platforms have increased the volume of data on the web. Further, proliferation in digital entertainment platforms such as Netflix, Hulu, and website-based services providing video streaming services have also increased the amount of video-based data in the web. It is imperative to note that video streaming service providers such as Netflix and others tend to use different formats and compression technologies to improve the user experience. As such, Web of Data is becoming more diversified despite most of the data relying on XML schema and improvements are necessary to ensure synergy between XML formats and SW technologies.

The differences between SW technologies and XML schema data models require usage of different semantics and query languages that render data retrieval difficult. However, it is prudent to develop tools and algorithms that can bridge the gap between SW technologies and XML schemas, and notably, it is not plausible and practical to convert all the pertinent XML schema data and legacy data in general to SW data (Borkum \& Frey, 2014; Chen, Chirkova, Sadri, \& Salo, 2013; Ishaq et al., 2013). Such is the limitation encountered in bridging the gap, and it is essential to consider making SW and XML schema interoperable and integrable to ensure smoother data query services over the internet. The objective of pursuing interoperability and integration between the two models is to ensure that users can access heterogeneous external data without having to worrying about the sources of the data as well as the format of the data ("Conference Proceedings of the European Society for Computing and Technology in Anaesthesia and Intensive Care (ESCTAIC) 13th Annual Meeting in co-operation with Institut fur Anasthesiologie, UniversitatsSpital Zurich,” 2002; Cordeiro \& de Carvalho, 2002; Cristea, 2004; Mika \& Akkermans, 2004; Payne \& Tamma, 2005; Uschold, 2003). As such, it is increasingly becoming essential to provide universal search query endpoints as opposed to legacy data in the context of Linked Data and both SW and XML data formats.

As such, the systematic review explored the mechanisms for exploiting legacy data in the context of Web of Data while representing and discussing the disparate features of SW and XML. In specific, the review compares, and contrasts SW and XML technologies studied between 2010 and 2018 with the objective of identifying trends in those standards and technologies. Of importance is the focus of relevant developments that different groups have achieved on SW technologies as well as XML-based schema. Most importantly, the systematic review addresses some of the emerging techniques for dealing with interoperability and integration gap between SW and XML data models (Batet et al., 2012; El-Mekawy, Östman, \& Hijazi, 2012; Tripathi, Gupta, \& Bhattacharya, 2012; Valkenhoef, Tervonen, Brock, \& Hillege, 2012). The exploration includes a detailed explanation of some of the emerging frameworks geared towards bridging the gap between the two data models. 


\section{NOVELTY AND CONTRIBUTION}

The PRISMA methodology has a meta-analysis component that requires focus on epidemiological research design. However, given that the research focused on interoperability and integration of SW and XML data models, it became apparent that the meta-analysis component of the PRISMA systematic review model was invalid in this research. Consequently, the systemic review used aspects such as title, the structure of the abstract, rational and objective(s) clarification in the introduction, information sources presented in the methods sections, synthesis of results and discussion, and relevance of the conclusion as the basis for including articles in the review. It is the criteria considered for article qualification under the auspices of the PRISMA systematic review model that makes the difference in the used approach. As for the research contribution of the paper, Web of Data and the linkage between SW and XML requires real-time interoperation and integration of the two data models to improve the usability of the web data considering the differences in data formats. As such, the systematic review accounts for technological advancements that aim at improving interoperability and integration between SW and XML schema-based data models. Given the growth rate of data, whether Web of Data or Big Data, it is apparent that future systems must move towards collaborative and interaction when it comes to data handling and exchange. Regarding such issues, creating a scholarly profile that explains and documents some of the current technologies for bridging the gap between SW and XML data models. Against this backdrop, it is critical to note the role that such interoperability and integration will play in the sharing of big data in the context of Web of Data.

The systematic review approach adopted in the paper focused relied on modified PRISMA checklist and DistillerSR review tool parameters and protocols. DistillerSR is a systematic review literature review system that Evidence Partners administer and develop to conduct systematic reviews based on some of the standard review standards. Besides adherence to the standard PRISMA requirements, the researcher made several modifications in some of the PRISMA checklist sections to meet the review requirements for the papers published on XML and SW technologies. The review relied on specific words and phrases for the search and the filters used in the study included publication period, type of paper (full text and scholarly), and narrowed to peer-reviewed papers. Given that several journals publish papers on the subject, ProQuest Central was the ideal database to search because of the access it has to several journals. The diversity of the search results is essential for improving the quality of the review because the sampling is more compressive than using a database that tends to focus on fewer number of journals.

As for the search strategy, the keywords used in the database included XML Schema, SW Technologies, XML and SW data models, and interoperability and integration of XML and SW models were the critical search strategies. It is critical to note that the focus was on XML and SW data models alongside the technologies for bridging the gap between XML and SW data models. Moreover, these two areas appear to be obscure with most research focusing on individual subtopics and disregarding the intersection and interoperation of the two models.

\section{RESULTS}

A preliminary search based on '(interoperability AND integration of XML AND SW models) AND (XML AND SW data models)' search string return over 200 documents but based on the DistillerSR review research, a majority of the documents failed to directly address the address either the comparison between XML and SWL or the interoperability and integration between these two models of web data presentation. However, modifying the research string and using XML and SW as the lead search phrase, a total of 50 articles turned up as suiting the search criteria. However, even with the filters, some of the articles did not address some of the emerging technologies critical in bridging the gap between SW and XML data formats. Figure 1 shows PRISMA the flowchart illustrates the search results and filters including qualified papers. The search results also consider an additional query implemented on Web of Science although most of the papers retrieved from the database are from computer-related journals found in ProQuest Central. 

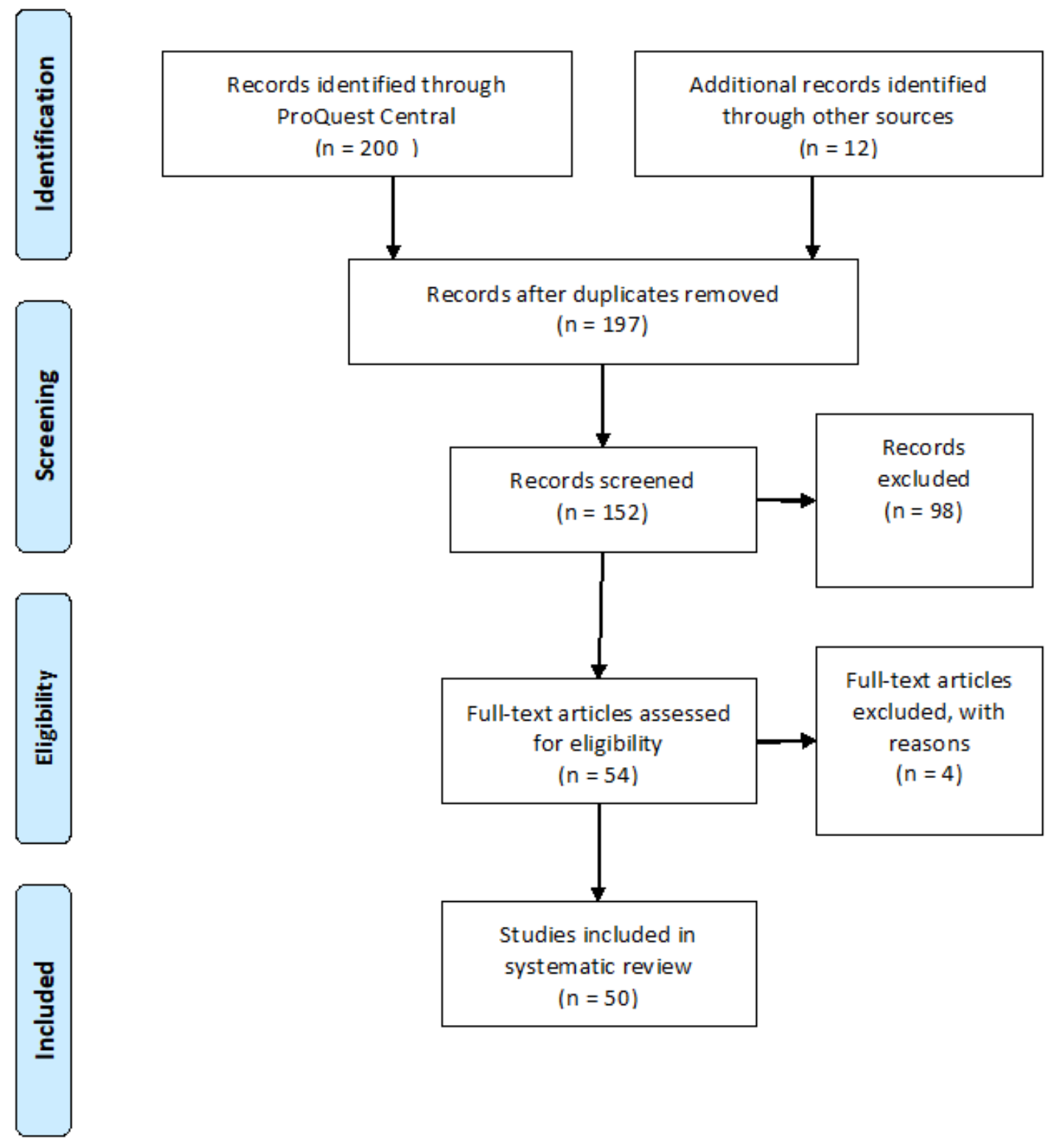

Figure 1. The PRISMA Framework and Article Analysis used in the Framework

\section{HISTORICAL AND TECHNICAL DISCOURSE OF XML AND SEMANTIC WEB}

Based on the previous discussion and results of the search strings, it is apparent that SW and XML models adopt and deploy different technologies. However, the differences observed and recorded between the two models are in three categories namely data, schema, and query levels. The standards that both XML and SW adopt at each of the three levels are different. For example, at the data level, XML models on XML data formats while SW uses RDF data format. At the schema level, XML model uses the XML schema while SW uses an RDF schema that leverages some of the features of OWL. Finally, at the query level, XML model uses XQuery while SW uses SPARQL. It suffices to note that XML is a data representation language in XML module while the RDF is a language for representing data in SW. The schema level describes the 
structure of the data both in XML and SW models with XML schema being the primary data model that the World Wide Web Consortium (W3C) recommends (Aleroud \& Karabatis, 2017; Ameri \& Patil, 2012; Kaur $\&$ Aggarwal, 2017). The number of articles published between 2010 and 2018 are distributed as follows.

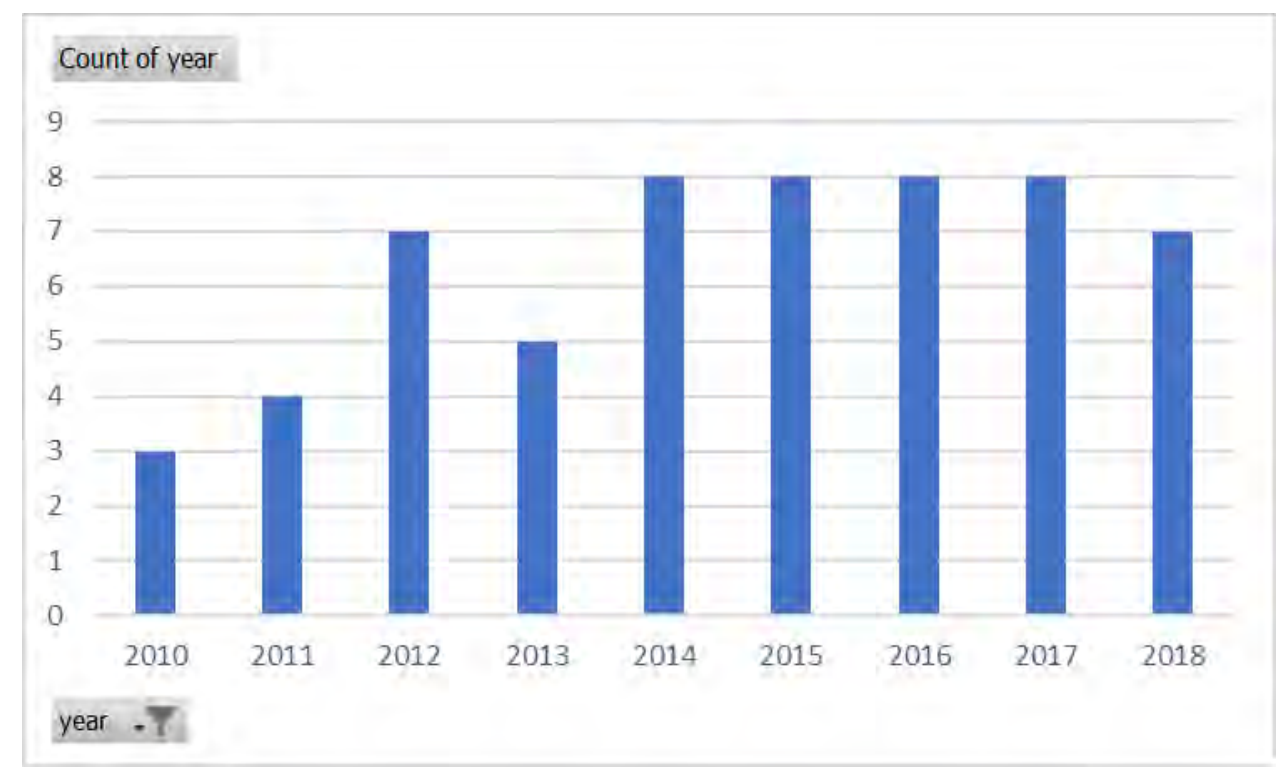

Figure 2. Distribution of the number of articles published on SW and XML between 2010 and 2018

As per Figure 2, the number of publications on SW and XML increased between 2010 and 2012, and after that between 2013 and 2014 before stagnating between 2014 and 2017. As per region distribution of the publications, the top three regions or more specifically cities leading in the publications include Heidelberg (13\%), Dordrecht (11\%), and Bradford (11\%) refer to Figure 3.

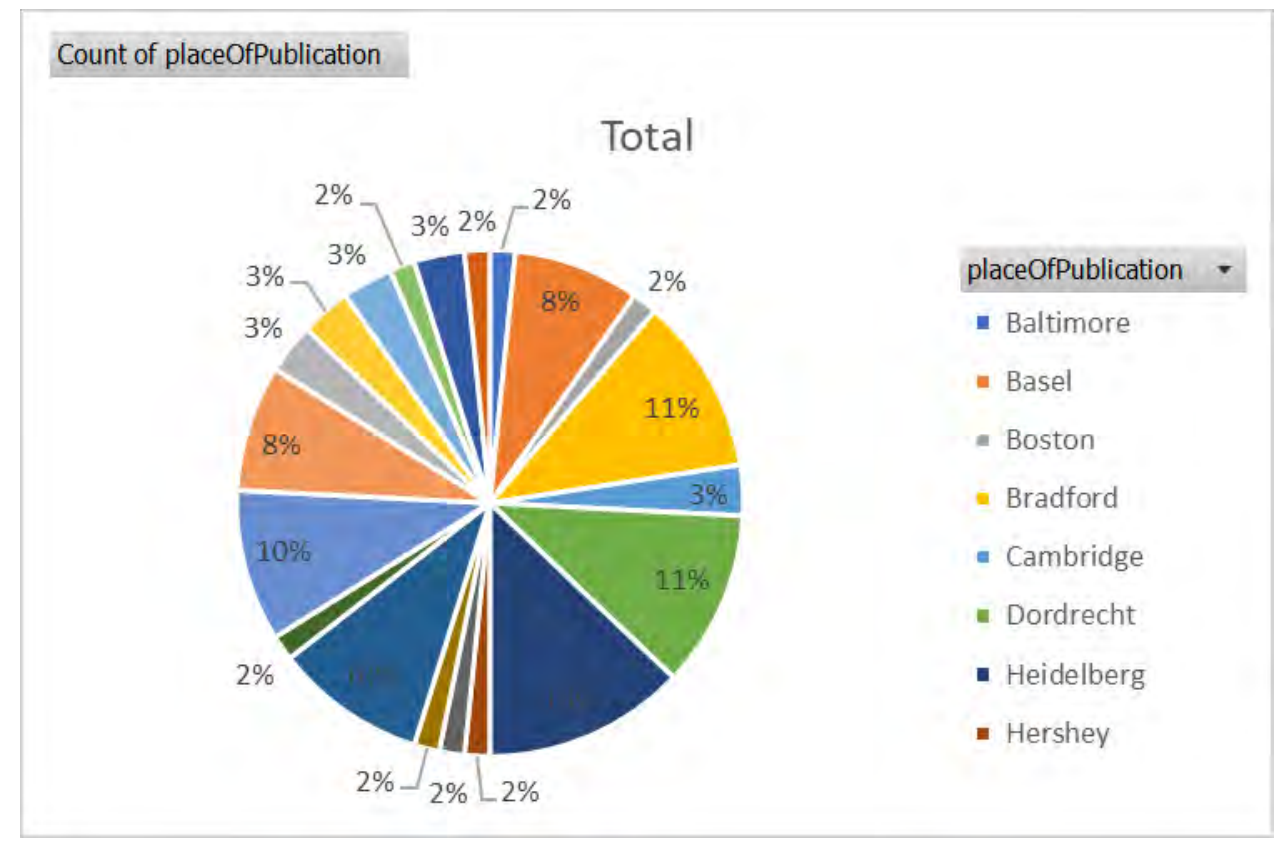

Figure 3. Distribution of Published Articles by Region 
However, it is imperative to note that XML Schema 1.0 is under development and W3C has plans to launch the framework to boost data sharing within partner programs in future. As for SW, both RDF schema and OWL describe the semantics and structure of the RDF data used in SW data query and analysis. The OWL-based SW consists of OWL validator, pellet, protégé four alpha (Figure 3). TopBraid composer and Jena. Each of these components performs a specific and distinct function and role in SW data dispersion and sharing technologies. As for the nature of the query level technologies and mechanisms, XML uses XQuery (XML Query Language to conduct advanced searches on XML datasets. On the other hand, SW uses SPARQL language alongside RDF query protocols as the standard language for querying RDF data. The current version of SPARQL has debugging and additional amendments and services on protocols, service descriptions, hypertext transfer protocols for managing RDF graphics and other critical regimes and functionalities. It was paramount to explore the fundamental principles of XML and SW as documented in the reviewed research papers. The evolution of both SW and XML technologies are as shown in Figure 4.

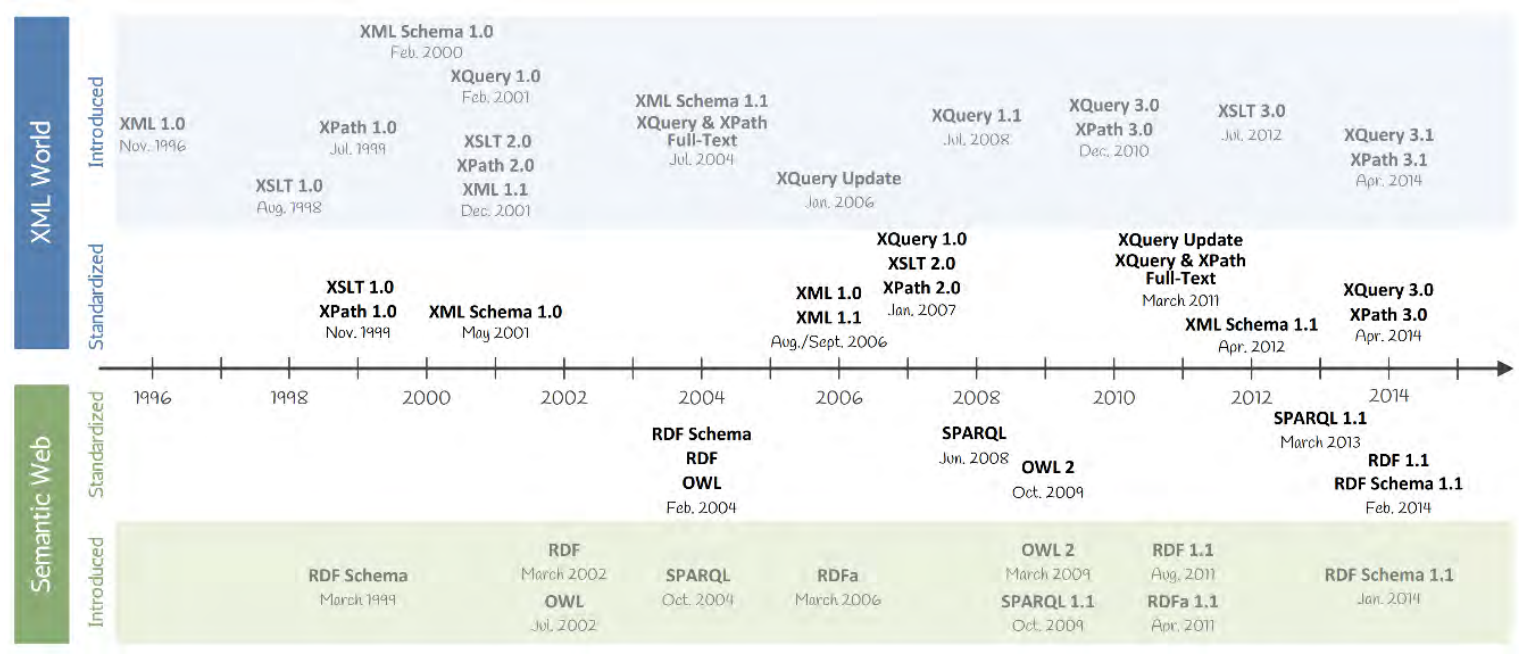

Figure 4. The Evolution of SW and XML Technologies between 2010 and 2018

\subsection{Data Level Comparisons and Contrasts}

As previously stated, the two data models exhibit obvious data level differences presented in the following discussions.

\subsubsection{Extensible Markup Language}

The is a representation of a document that in essence is a template or an outline for preparing documents based on the XML standard. It uses the same tags used in Hypertext Markup Language (HTML) although the XML tags lack restrictive tag and interpretive semantics. As such users can define and personalise tags to meet their document formatting and output needs. Hence, XML consists of a plain text that contains the content of the document and a markup that is an equivalence of a cascading styling sheet used in format and style HTML documents (Askarunisa, Punitha, \& Ramaraj, 2011; Isern et al., 2011; Kovacevic, Ivanovic, Milosavljevic, Konjovic, \& Surla, 2011; Nowling et al., 2011). The XML language uses tags that assume the tree-branch pattern. It is imperative to note that XML has distinct and standard elements including but not limited to nodes of the document, elements, comments, specs for namespaces, and instructions among others. The XML document structure has unique and standard features similar to those found in document processing languages such as Latex. A majority the data in the web are based on this data format, and due its importance and role in data available, several web data scrapping methods and tools have been devised to aid in the process of extracting, transforming and loading such data onto suitable analytical platforms. 


\subsubsection{Resource Description Framework}

The RDF is also a general-purpose language that represents web information resources but on the semantic web side. In comparison to XML, the RDF is a lightweight language that models data using central RDF statements and concepts. It is flexible and simple to use because of its reliance on central RDF model concept that requires a subject, a predicate, and an object to create data nodes that users can query. In the context of RDF statement, the subject refers to the resources that the web data is describing while the predicate points towards a specific property of the object. The object in question must have a value, and it is imperative to note that the subject cannot be an Internationalized Resource Identifier (IRI) or a blank node. Conversely, it is mandatory for a predicate to an IRI while the object can either be a blank node, an IRI, or an RDF literal. Most importantly, graphically, RDF statements with clear labels are graphs with resources serving as the nodes and the statements forming the arcs in the resultant graphs(Dallas, 2016; Drewes, Kuglitsch, Adám, \& Rózsa, 2016; Souag, Mazo, Salinesi, \& Comyn-wattiau, 2016). Ideally, the arcs that the statements form connect subject and object nodes, and the graph in its entirety is the RDF Dataset.

\subsection{Schema Level Comparisons and Contrasts}

At the schema level, XML, RDF and OWL tend to exhibit distinct competitive features deduced from their functional differences. Some of the features and the differences observed from the systematic review are discussed in the following subtopic.

\subsubsection{Extensible Markup Language Schema}

The XML schema is a language schema that W3C developed using the XML syntax, and it describes the structure and format of all documents written in XML (Robson \& Boray, 2015; Soylu, Giese, Jimenez-ruiz, Vega-gorgojo, \& Horrocks, 2016). From a technical perspective, the XML syntax has rich capabilities that render one of the commonly used formats for web data. In practical applications, the XML schema specifies the specific element hierarchy including all data type constraints available as options within predefined options of the data. The optional constraints restrict the number of occurrences of the number as well as the nature of attributes.

A standard XML document consists of components that have root as the delimiting factor at the beginning and end of the said document. The delimiters are tags that specify the type of XML schema, and the tags are either simple or complex depending on the nature of the tags that the model uses. It is important to draw the similarities in syntax between XML schema and other programming languages regarding data types. That is, XML uses standard data syntax such as string, integer, and double among others. Furthermore, XML schema also has complex data types represented as classes and constructs that consist of features and attributes of desired elements of the resultant document.

As for the structure of the document, XL schema has rich structuring functionalities. In most cases, the schema document has a more or less or preprogrammed structure serving as a template based on specified sequences or unordered elements created using choice and all tag commands. Therefore, it is critical to understand that OWL has constructs assigned unique names and nested accordingly to manage and handle unnamed and unstructured data. However, it is imperative to note that unstructured and complex attributes may require referencing using appropriate attributes to avoid cyclic redundancies that would other derail the performance of the XML extraction process. Based on the information available on the OWL website in conjunction with the updates that $\mathrm{W} 3 \mathrm{C}$ provides, OWL has two versions, and the second version has high in-memory efficiency for implemented complex references.

The XML schema 1.1 is compatible with Schema 1.0 although it introduced the alternative element that allows a user to have and specify a set of data types. The feature allows the schema to circumvent some of the dataset restriction it faces in the previous versions and the XML Schema 1.1 has a redefine function for recalibrating variables that do not conform to the limits predetermined in the options of the schema. Furthermore, XML Schema 1.1 has error management features, union capabilities for the value spaces of primitive attributes, and timestamp for tracing the time series associated with each of the attributes. Currently, XML Schema has reached its third version and an example of a document based on the XML Schema 3.0 and above id the Europass CV. The latest version the schema is XML Schema 3.2 that uses a top-down approach in handling its elements as well as additional elements such as key, imports, and attribute types (Burke, 2009; Torre, 2009). It is also imperative to note that the latest version of the schema has a wide variety of data types and the expansion accommodates some of the emerging complex data fields. 


\subsubsection{RDF Schema}

The schema leverages the concept of RDF, and it builds vocabulary, and subsequent dictionary referred to as RDFS based on its relationship between the vocabulary library and the resources available within the referenced libraries and packages. As per fundamentals of RDF schema, such packages belong to instance class and the property value associated with confirms the relationship. It is pertinent to note that it is possible to rearrange XML data classes into based in hierarchical structures that are subparts of objects and properties. All the RDFs classes correspond to the concept type of categorisation of a given attribute. Of importance is to note that the function and characteristics of RDF stagnated regardless of the evolution in some aspects of the framework. Regardless of the similarities between the two languages, OWL is richer hence superior to RDF, and the basic difference is evident in the definition of OWL class construct as opposed to that of RDF.

\subsubsection{Web Ontology Language}

The OWL is the standard ontology for web data and content, and it is similar to the RDF. It has two versions with innumerable updates, but the latest introduction involved the inclusion of a temporal ontology element in an OWL. The latest edition focuses on the characteristics of temporal concepts that account and focus on the description of temporal properties of the resources used in WWW pages. It is also critical to recognise that OWL 2.0 and the latest versions (Löhe \& Legner, 2010; Russomanno, Chari, \& Halford, 2008; Sundberg, Sundberg, \& Schofield, 2008). Despite the improvement between the versions of the frameworks, it is critical to note that most upgrades involved improvements that had little changes to do with the overall functionality of the framework. Besides modal differences, there is a difference observed based on concrete syntax, semantics, basic constructs, identity constraints, and user-defined data types and attributes.

\subsection{Query Level Comparisons}

The contrasts drawn at query level are the basis for exploring the differences between SW and XML data models.

\subsubsection{XQuery and XPath}

Given the differences between SW and XML data formats, it is prudent to explore query techniques that can address the gap between the two technologies. The current XML schema depends on XML Path Language to sect document nodes within the targeted XML dataset. The XPath query language is a component of the XQuery language and it has a tree-like structure for displaying the query results and all XML documents retrieved in the process(Fox \& Kozyra, 2015; Ilie-Zudor et al., 2015; Kardan, Aziz, \& Shahpasand, 2015; Ratnam et al., 2014; Robson, Caruso, \& Balis, 2015). The XQuery has many features, but none of which addresses the gap between SW and XML and SPARQL has emerged as a technique of interest in bridging the gap.

Besides the security concern and mandate of $\mathrm{W} 3 \mathrm{C}$, one of the key concern areas is the advancement of and integration of crucial web data and communication technologies. SPARQL is the future solution to addressing this problem and future considerations ought to consider the influence and impact of SPARQL on the future of interoperability and integration of SW and XML data models(Hung et al., 2007; Paquette, 2007; Rasseneur-Coffinet, Smyrniou, \& Tchounikine, 2007; Soroor \& Tarokh, 2006). Regardless of the version of SPARQL, one of its major features is the support for data retrieval between these two data formats, and the key functionality and benefit of using SPARQL is the full range of query options including the options to select, construct, ask, and describe the construct of interest. As an operational principle, SPARQL executes a select query that returns an amalgamated sequence of output with restricted variable binding. Further, the results of the ask query is a Boolean response assuming either a value of yes or no. Most importantly, the most recent versions of SPARQL contains RDF modules with graphing and reporting capabilities for various solutions. 


\section{CONCLUSION}

The opportunities for the application of SPARQL and other related interoperability and integration techniques are endless because Web of Data continues to grow, and the complexity of the wed data will require seamless integration between SW and XML data formats and related technology. The fact is that technologies such SPARQL are playing a crucial role in ensuring that SW and XML data are receivable with ease. However, it is pertinent to highlight that the search articles also captured and accounted for articles that are not necessarily addressing the area of interest and it would be prudent to conduct a survey target studies that have explored the interoperability and integration of SW and XML schemas and data formats.

\section{REFERENCES}

Aleroud, A., \& Karabatis, G. (2017). Contextual information fusion for intrusion detection: a survey and taxonomy. Knowledge and Information Systems, 52(3), 563-619. https://doi.org/http://dx.doi.org/10.1007/s10115-017-1027-3

Ameri, F., \& Patil, L. (2012). Digital manufacturing market: a semantic web-based framework for agile supply chain $\begin{array}{lllll}\text { deployment. Journal of Intelligent } & \text { Manufacturing, } & \text { 23(5), }\end{array}$ https://doi.org/http://dx.doi.org/10.1007/s10845-010-0495-z

Anonymous. (2014). Engineering \& Physical Sciences in Medicine Conference 2013 Pan Pacific Hotel, Perth, Australia, 3-7 November 2013 Oral presentation abstracts. Australasian Physical \& Engineering Sciences in Medicine, 37(1), 153-269. https://doi.org/http://dx.doi.org/10.1007/s13246-014-0248-y

Askarunisa, A., Punitha, K. A. J., \& Ramaraj, N. (2011). Test Case Generation and Prioritization for Composite Web Service Based on OWL-S. Neural Network World, 21(6), 519-537. Retrieved from https://search.proquest.com/docview/1010622274?accountid=145382

Batet, M., Isern, D., Marin, L., Martínez, S., Moreno, A., Sánchez, D., ... Gibert, K. (2012). Knowledge-driven delivery of home care services. Journal of Intelligent Information Systems, 38(1), 95-130. https://doi.org/http://dx.doi.org/10.1007/s10844-010-0145-0

Batra, S., Sachdeva, S., \& Bhalla, S. (2018). Entity Attribute Value Style Modeling Approach for Archetype Based Data. Information, 9(1), 2. https://doi.org/http://dx.doi.org/10.3390/info9010002

Borkum, M. I., \& Frey, J. G. (2014). Usage and applications of Semantic Web techniques and technologies to support chemistry research. Journal of Cheminformatics, 6. https://doi.org/http://dx.doi.org/10.1186/1758-2946-6-18

Burke, M. (2009). The semantic web and the digital library. Aslib Proceedings, 61(3), $316-322$. https://doi.org/http://dx.doi.org/10.1108/00012530910959844

Chen, D., Chirkova, R., Sadri, F., \& Salo, T. J. (2013). Query optimization in information integration. Acta Informatica, 50(4), 257-287. https://doi.org/http://dx.doi.org/10.1007/s00236-013-0179-1

Conference Proceedings of the European Society for Computing and Technology in Anaesthesia and Intensive Care (ESCTAIC) 13th Annual Meeting in co-operation with Institut fur Anasthesiologie, UniversitatsSpital Zurich. (2002). Journal of Clinical Monitoring and Computing, 17(7-8), 467. Retrieved from https://search.proquest.com/docview/217133632?accountid=145382

Cordeiro, M. I., \& de Carvalho, J. (2002). Web services: What they are and their importance for libraries. VINE, 32(4), 46-62. https://doi.org/http://dx.doi.org/10.1108/03055720210471148

Cristea, A. I. (2004). What can the Semantic Web do for Adaptive Educational Hypermedia? Journal of Educational $\begin{array}{llllll}\text { Technology \& } & \text { Society, } & 7(4), & 40-58 . & \text { Retrieved } & \text { from }\end{array}$ https://search.proquest.com/docview/1287054280?accountid=145382

Dallas, C. (2016). Digital curation beyond the "wild frontier": a pragmatic approach. Archival Science, 16(4), 421-457. https://doi.org/http://dx.doi.org/10.1007/s10502-015-9252-6

Drewes, H., Kuglitsch, F., Adám, J., \& Rózsa, S. (2016). The Geodesist's Handbook 2016. Journal of Geodesy, 90(10), 907-1205. https://doi.org/http://dx.doi.org/10.1007/s00190-016-0948-Z

El-Mekawy, M., Östman, A., \& Hijazi, I. (2012). A Unified Building Model for 3D Urban GIS. ISPRS International Journal of Geo-Information, 1(2), 120-145. https://doi.org/http://dx.doi.org/10.3390/ijgi1020120

Fox, P., \& Kozyra, J. (2015). eScience and Informatics for international science programs. Progress in Earth and Planetary Science, 2(1), 1-9. https://doi.org/http://dx.doi.org/10.1186/s40645-015-0042-9

Grossi, V., Romei, A., \& Turini, F. (2017). Survey on using constraints in data mining. Data Mining and Knowledge Discovery, 31(2), 424-464. https://doi.org/http://dx.doi.org/10.1007/s10618-016-0480-z 
Hung, P. C. K., Chiu, D. K. W., Fung, W. W., Cheung, W. K., Wong, R., Choi, S. P. M., ... Cheng, V. S. Y. (2007). End-to-end privacy control in service outsourcing of human intensive processes: A multi-layered Web service integration approach. Information Systems Frontiers, 9(1), 85-101. https://doi.org/http://dx.doi.org/10.1007/s10796006-9019-y

Ilie-Zudor, E., Ekárt, A., Kemeny, Z., Buckingham, C., Welch, P., \& Monostori, L. (2015). Advanced predictive-analysis-based decision support for collaborative logistics networks. Supply Chain Management, 20(4), 369-388. Retrieved from https://search.proquest.com/docview/1691011014?accountid=145382

Isern, D., Moreno, A., Sánchez, D., Hajnal, Á., Pedone, G., \& Varga, L. Z. (2011). Agent-based execution of personalised home care treatments. Applied Intelligence, 34(2), 155-180. https://doi.org/http://dx.doi.org/10.1007/s10489-009-0187-6

Ishaq, I., Carels, D., Teklemariam, G. K., Hoebeke, J., Abeele, F. Vanden, Poorter, E. De, ... Demeester, P. (2013). IETF Standardization in the Field of the Internet of Things (IoT): A Survey. Journal of Sensor and Actuator Networks, 2(2), 235-287. https://doi.org/http://dx.doi.org/10.3390/jsan2020235

Jing, X., Kay, S., Marley, T., \& Hardiker, N. R. (2014). Integration of an OWL-DL Knowledge Base with an EHR Prototype and Providing Customized Information. Journal of Medical Systems, 38(9), 1-75. https://doi.org/http://dx.doi.org/10.1007/s10916-014-0075-4

Kardan, A. A., Aziz, M., \& Shahpasand, M. (2015). Adaptive systems: a content analysis on technical side for e-learning environments. The Artificial Intelligence Review, 44(3), 365-391. https://doi.org/http://dx.doi.org/10.1007/s10462015-9430-1

Kaur, N., \& Aggarwal, H. (2017). Evaluation of Information Retrieval Based Ontology Development Editors for Semantic Web. International Journal of Modern Education and Computer Science, 9(7), 63. https://doi.org/http://dx.doi.org/10.5815/ijmecs.2017.07.07

Kovacevic, A., Ivanovic, D., Milosavljevic, B., Konjovic, Z., \& Surla, D. (2011). Automatic extraction of metadata from scientific publications for CRIS systems. Program, 45(4), 376-396. https://doi.org/http://dx.doi.org/10.1108/00330331111182094

Li, X., Martínez, J.-F., \& Rubio, G. (2017). Towards a Hybrid Approach to Context Reasoning for Underwater Robots. Applied Sciences, 7(2), 183. https://doi.org/http://dx.doi.org/10.3390/app7020183

Löhe, J., \& Legner, C. (2010). SOA adoption in business networks: do service-oriented architectures really advance inter-organizational integration? Electronic $181-196$. https://doi.org/http://dx.doi.org/10.1007/s12525-010-0046-7

MIKA, P., \& AKKERMANS, H. (2004). Towards a new synthesis of ontology technology and knowledge management. The Knowledge Engineering Review, 19(4), 317-345. Retrieved from https://search.proquest.com/docview/217528594?accountid=145382

Naumenko, A., Nikitin, S., Terziyan, V., \& Zharko, A. (2005). Strategic industrial alliances in paper industry: XML- vs Ontology-based integration platforms. The Learning Organization, 12(5), $492-514$. https://doi.org/http://dx.doi.org/10.1108/09696470510611438

Nowling, R. J., Vyas, J., Weatherby, G., Fenwick, M. W., Ellis, H. J. C., \& Gryk, M. R. (2011). CONNJUR spectrum translator: an open source application for reformatting NMR spectral data. Journal of Biomolecular NMR, 50(1), 83-89. https://doi.org/http://dx.doi.org/10.1007/s10858-011-9497-1

Paquette, G. (2007). An Ontology and a Software Framework for Competency Modeling and Management. Journal of Educational Technology \& Society, 10(3), $1-21$. Retrieved from https://search.proquest.com/docview/1287039622?accountid=145382

PAYNE, T., \& TAMMA, V. (2005). Towards Semantic Web agents: Knowledge Web and AgentLink. The Knowledge Engineering Review, 20(2), Retrieved 191-196. https://search.proquest.com/docview/217507142?accountid=145382

Rasseneur-Coffinet, D., Smyrniou, G., \& Tchounikine, P. (2007). Supporting Learners' Appropriation of a Web-Based Learning Curriculum. International Journal of Distance Education Technologies, 5(4), 46-79. https://doi.org/http://dx.doi.org/10.4018/jdet.2007100104

Ratnam, J., Zdrazil, B., Digles, D., Cuadrado-Rodriguez, E., Neefs, J.-M., Tipney, H., ... Chichester, C. (2014). The Application of the Open Pharmacological Concepts Triple Store (Open PHACTS) to Support Drug Discovery Research. PLoS One, 9(12). https://doi.org/http://dx.doi.org/10.1371/journal.pone.0115460

Robson, B. (2016). Studies in using a universal exchange and inference language for evidence based medicine. Semi-automated learning and reasoning for PICO methodology, systematic review, and environmental epidemiology. $\begin{array}{lllll}\text { Computers in } & \text { Biology } & \text { Medicine, } & 79, & 299-323 .\end{array}$ https://doi.org/http://dx.doi.org/10.1016/j.compbiomed.2016.10.009 
Robson, B., \& Boray, S. (2015). Implementation of a web based universal exchange and inference language for medicine: Sparse data, probabilities and inference in data mining of clinical data repositories. Computers in Biology and Medicine, 66, 82-102. https://doi.org/http://dx.doi.org/10.1016/j.compbiomed.2015.07.015

Robson, B., Caruso, T. P., \& Balis, U. G. J. (2015). Suggestions for a web based universal exchange and inference language for medicine. Continuity of patient care with PCAST disaggregation. Computers in Biology and Medicine, 56, 51-66. https://doi.org/http://dx.doi.org/10.1016/j.compbiomed.2014.10.022

Russomanno, D., Chari, S., \& Halford, C. (2008). Sparse Detector Imaging Sensor with Two-Class Silhouette Classification. Sensors, 8(12), 7996-8015. https://doi.org/http://dx.doi.org/10.3390/s8127996

Scharffe, F., Zamazal, O., \& Fensel, D. (2014). Ontology alignment design patterns. Knowledge and Information Systems, 40(1), 1-28. https://doi.org/http://dx.doi.org/10.1007/s10115-013-0633-y

Soroor, J., \& Tarokh, M. J. (2006). Innovative SCM. VINE, 36(3), 304-340. https://doi.org/http://dx.doi.org/10.1108/03055720610703597

Souag, A., Mazo, R., Salinesi, C., \& Comyn-wattiau, I. (2016). Reusable knowledge in security requirements engineering: a systematic mapping study. Requirements Engineering, 21(2), 251-283. https://doi.org/http://dx.doi.org/10.1007/s00766-015-0220-8

Soylu, A., Giese, M., Jimenez-ruiz, E., Kharlamov, E., Zheleznyakov, D., \& Horrocks, I. (2017). Ontology-based end-user visual query formulation: Why, what, who, how, and which? Universal Access in the Information Society, 16(2), 435-467. https://doi.org/http://dx.doi.org/10.1007/s10209-016-0465-0

Soylu, A., Giese, M., Jimenez-ruiz, E., Vega-gorgojo, G., \& Horrocks, I. (2016). Experiencing OptiqueVQS: a multi-paradigm and ontology-based visual query system for end users. Universal Access in the Information Society, 15(1), 129-152. https://doi.org/http://dx.doi.org/10.1007/s10209-015-0404-5

Sundberg, J. P., Sundberg, B. A., \& Schofield, P. (2008). Integrating mouse anatomy and pathology ontologies into a phenotyping database: Tools for data capture and training. Mammalian Genome, 19(6), 413-419. https://doi.org/http://dx.doi.org/10.1007/s00335-008-9123-z

Torre, I. (2009). Adaptive systems in the era of the semantic and social web, a survey. User Modeling and User - Adapted Interaction, 19(5), 433-486. https://doi.org/http://dx.doi.org/10.1007/s11257-009-9067-3

Tripathi, R., Gupta, M. P., \& Bhattacharya, J. (2012). Interoperability adoption among government and corporate portals in India: a study. Journal of Enterprise Information Management, 25(2), 98-122. https://doi.org/http://dx.doi.org/10.1108/17410391211204374

Umar, A. (2005). IT Infrastructure to Enable Next Generation Enterprises. Information Systems Frontiers, 7(3), 217. https://doi.org/http://dx.doi.org/10.1007/s10796-005-2768-1

Uschold, M. (2003). Where are the semantics in the semantic web? AI Magazine, 24(3), 25-36. Retrieved from https://search.proquest.com/docview/208125637? accountid=145382

Valkenhoef, G. van, Tervonen, T., Brock, B. de, \& Hillege, H. (2012). Deficiencies in the transfer and availability of clinical trials evidence: a review of existing systems and standards. BMC Medical Informatics and Decision Making, 12, 95. https://doi.org/http://dx.doi.org/10.1186/1472-6947-12-95

Zemmouchi-Ghomari, L. (2018). Cohabitation of Relational Databases and Domain Ontologies in the Semantic Web Context. Journal of Systems Integration, 9(1), 42-57. Retrieved from https://search.proquest.com/docview/2000969229?accountid=145382 